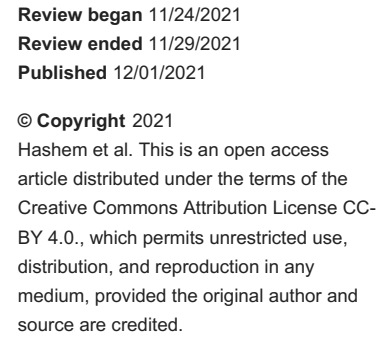

\section{Thrombosis and Anticoagulant Therapy Among Pediatric Cancer Patients: Real-Life Data}

\author{
Hasan Hashem ${ }^{1}$, Momen Zeineddin ${ }^{1}$, Rayan Bater ${ }^{2}$, Nisreen Amayiri ${ }^{1}$, Wiam Al Qasem ${ }^{3}$, Bilasan
} Hammo $^{1}$, Iyad Sultan ${ }^{1}$, Rama AlMasri ${ }^{4}$, Hikmat Abdel-Razeq ${ }^{2,5}$

1. Department of Pediatrics, King Hussein Cancer Center, Amman, JOR 2. Department of Internal Medicine, King Hussein Cancer Center, Amman, JOR 3. Department of Pharmacy, King Hussein Cancer Center, Amman, JOR 4. Department of Internal Medicine, Istishari Hospital, Amman, JOR 5. Department of Medicine, School of Medicine, University of Jordan, Amman, JOR

Corresponding author: Hikmat Abdel-Razeq, habdelrazeq@khcc.jo

\title{
Abstract
}

Background: Venous thromboembolism (VTE) in children is relatively rare, and more so among those with cancer. In this study, we report the characteristics and outcomes of children with cancer-associated thrombosis.

Methods: We reviewed institutional databases for all children with cancer and a diagnosis of VTE at King Hussein Cancer Center in Jordan. Variables reviewed are patients' clinical characteristics, treatment for cancer, and anticoagulation therapy.

Results: Between January 2011 and December 2018, a total of 45 patients fulfilled the inclusion criteria, and the median age was $10.4(0.8-17.9)$ years. The most common underlying diagnosis was acute lymphoblastic leukemia ( $\mathrm{n}=13,29 \%)$. At the time of VTE, 29 (64.4\%) patients were receiving chemotherapy, and eight (17.8\%) had a central venous catheter (CVC). The majority of patients $(n=37,82 \%)$ developed VTE within 30 days of hospitalization. Thrombosis mostly involved the extremities $(n=23,51 \%)$ and sagittal vein $(n=12$, 26.7\%). All patients were treated with low-molecular-weight heparin (LMWH), complicated by bleeding in three $(6.6 \%)$ patients.

Conclusion: In contrast to adults, VTE in pediatric cancer patients is more associated with chemotherapy and recent hospitalization. LMWH is a safe and effective therapy for children with cancer who develop VTE.

Categories: Pediatrics, Oncology, Hematology

Keywords: thromboprophylaxis, anticoagulation, venous thromboembolism, pediatrics thrombosis, cancer

\section{Introduction}

Venous thromboembolism (VTE), including deep vein thrombosis (DVT) or pulmonary embolism (PE), is a known complication of malignancy [1]. The majority of studies investigating incidence, risk factors, and treatment of VTE in the setting of cancer have focused on the adult population [2,3]. While VTE is rare in children with cancer, it has been increasingly diagnosed [4-8].

Children with cancer are at increased risk of VTE compared with the general pediatric population. The incidence of VTE in children with cancer ranges from 2.0 to $16.0 \%[9,10,11]$. VTE in children with cancer is multifactorial and includes disease-related factors (type of cancer, inflammation, and abnormal blood flow) and treatment-related factors (presence of central venous catheters [CVC], surgery, and type of chemotherapy) $[12,13]$.

The majority of data on the treatment of VTE in children with cancer has been extrapolated from adults literature and observational studies in the general pediatric population. Nevertheless, there have been recent guidelines from the Children's Oncology Group and the American Society of Hematology specific to children with cancer, and low-molecular-weight heparin (LMWH) is the anticoagulant of choice [14]. The significant burden of VTE complications, including potential worsened prognosis, seems to justify that certain patients with cancer and high-risk features may be considered for primary thromboprophylaxis. However, to date, there have been no clear guidelines in pediatric cancer patients with regard to thromboprophylaxis. In addition, many pediatric oncologists are hesitant to use prophylactic anticoagulation in children with cancer because of the associated risk of bleeding, mostly due to pre-existing thrombocytopenia [15].

The majority of information on VTE in children with cancer originates from studies conducted in children with acute lymphoblastic leukemia (ALL) $[9,16]$. Other studies evaluated VTE in pediatric patients with various oncologic diagnoses [10,17-19]. Hence, we conducted a retrospective study to evaluate the characteristics and treatment of VTE among pediatric patients with cancer at King Hussein Cancer Center, 


\section{Cureus}

Amman, Jordan.

This article was previously presented as a poster at the 2020 ISTH Virtual Congress on July 11, 2020.

\section{Materials And Methods}

This is a retrospective single-center study. Medical records and electronic institutional and pharmacy databases were reviewed for all children $<18$ years of age, with a diagnosis of cancer, who received any form of anticoagulation. We included pediatric patients with cancer and VTE. All VTE events were radiologically confirmed. Variables collected include patients' age, gender, primary cancer type, type of anticancer therapies (surgery, chemotherapy, and radiotherapy) in addition to type and duration of anticoagulation used, and associated complications. Descriptive statistics were performed for all variables. Continuous variables with normal distribution were presented as mean (standard deviation [SD]); non-normal variables were described as median (interquartile range [IQR]), and categorical variables were expressed as numbers (percentages). Results for continuous variables are expressed as median (range), and categorical variables are expressed as numbers (percentage). This study was approved by the Institutional Review Board (IRB) at King Hussein Cancer Center (KHCC) (approval number: 18-KHCC-107).

\section{Results}

From January 2011 to December 2018, 45 eligible pediatric patients with cancer and radiologically confirmed VTE were identified and included in our study. All were younger than 18 years at the time of cancer diagnosis. The median age at VTE diagnosis was 10.4 (range 0.8-17.9) years, and 22 (48.9\%) were males. The patients' characteristics are detailed in Table 1.

\begin{tabular}{|c|c|c|}
\hline Characteristics & Number & Percentage \\
\hline \multicolumn{3}{|l|}{ Age (years) } \\
\hline Median (range) & \multicolumn{2}{|l|}{$10.4(0.8-17.9)$} \\
\hline \multicolumn{3}{|l|}{ Primary tumor } \\
\hline Leukemia & 13 & 28.9 \\
\hline Lymphoma & 6 & 13.3 \\
\hline Bone & 6 & 13.3 \\
\hline Brain & 6 & 13.3 \\
\hline RMS & 3 & 6.7 \\
\hline Others & 11 & 24.5 \\
\hline Chemotherapy & 40 & 88.9 \\
\hline Radiotherapy & 3 & 6.7 \\
\hline Anticoagulation prophylaxis & 0 & 0 \\
\hline Surgery & 7 & 15.5 \\
\hline
\end{tabular}

TABLE 1: Patients characteristics $(n=45)$

RMS, Rhabdomyosarcoma

\section{Risk factors for VTE}

The median time from diagnosis of cancer to VTE was 30 (1-3333) days. Most $(\mathrm{n}=37,82.2 \%)$ patients were hospitalized within 30 days of VTE occurrence. Eight (17.7\%) patients had CVC, seven (15.5\%) had major surgery, and three (6.7\%) had radiation therapy before VTE diagnosis. Twenty-nine (64.4\%) patients had received chemotherapy within one month of VTE; 23 (79.3\%) of them developed VTE during the first month of chemotherapy.

\section{Site of VTE}

The most common site for VTE formation was the lower limbs $(n=21,46.6 \%)$, followed by cerebral sagittal sinus venous thrombosis (CSVT) $(n=12,26.6 \%)$. Two patients developed upper extremity DVT. PE was 


\section{Cureus}

diagnosed in four patients, two of which had lower extremity VTE (Table 2).

\begin{tabular}{|c|c|c|}
\hline Clinical Variables & Number of Patients & Percentage \\
\hline VTE within 30 days of last chemotherapy & 29 & 64.4 \\
\hline VTE within 30 days of admission & 37 & 82.2 \\
\hline \multicolumn{3}{|l|}{ VTE Site } \\
\hline Lower extremity & $21^{*}$ & 46.7 \\
\hline Upper extremity & 2 & 4.4 \\
\hline PE & $4^{*}$ & 8.8 \\
\hline CSVT & 12 & 26.7 \\
\hline Others & 8 & 17.8 \\
\hline \multicolumn{3}{|l|}{ Complications } \\
\hline Minor bleeding & 1 & 2.2 \\
\hline Major bleeding & 2 & 4.4 \\
\hline Thrombocytopenia & 14 & 31.1 \\
\hline None & 28 & 62.3 \\
\hline
\end{tabular}

\section{TABLE 2: Venous thromboembolism (VTE) characteristics}

${ }^{*}$ Two patients developed both lower extremity DVT and PE.

CSVT, Cerebral sagittal sinus venous thrombosis; PE, pulmonary embolism; DVT, deep vein thrombosis.

CSVT was diagnosed at a median age of 11.7 (2.2-16.7) years. Of the 12 patients with CSVT, five (41.6\%) had B-ALL, three (25.0\%) had a brain tumor, while Hodgkin lymphoma and synovial sarcoma were diagnosed in two patients (Table 3). Nine (75.0\%) of 12 patients were on steroids at the time of VTE diagnosis. All B-ALL patients received steroids and asparaginase and were either on induction or consolidation chemotherapy. 


\section{Cureus}

\begin{tabular}{|c|c|c|}
\hline Characteristics & Number & Percentage \\
\hline \multicolumn{3}{|l|}{ Age (years) } \\
\hline Median (range) & \multicolumn{2}{|l|}{$11.7(2.2-16.7)$} \\
\hline \multicolumn{3}{|l|}{ Primary tumor } \\
\hline Leukemia & 5 & 41.6 \\
\hline Brain & 3 & 25 \\
\hline Hodgkin lymphoma & 2 & 16.7 \\
\hline Synovial sarcoma & 2 & 16.7 \\
\hline Chemotherapy & 11 & 91.7 \\
\hline Radiotherapy & 1 & 8.3 \\
\hline Anticoagulation prophylaxis & 0 & 0 \\
\hline Surgery & 3 & 25.0 \\
\hline
\end{tabular}

\section{TABLE 3: Clinical characteristics of patients with CSVT $(n=12)$}

\section{Treatment of VTE}

All VTEs were treated with Enoxaparin at a dose of $1 \mathrm{mg} / \mathrm{kg} / \mathrm{dose}$ twice daily, and none were on thromboprophylaxis before the diagnosis of VTE. Anti-Xa measurements are not available at our institution, and hence, all patients remained on the same dose of Enoxaparin throughout the treatment duration. Duration of anticoagulation varied from three months in 21 (46.6\%) patients, six months in nine (20\%) patients, and 12 months in 13 (28.8\%) patients (Table 2). Three patients (6.7\%) developed bleeding as a complication of anticoagulation; two were major, and all have recovered. Recurrent VTE was encountered in two patients, and post-thrombotic syndrome was reported in one patient.

\section{Discussion}

In this retrospective study, we highlight a real-life experience addressing the issues related to diagnoses, treatment, and associated complications encountered in pediatric cancer patients over an eight-year period.

Leukemia was the most common cancer among pediatric patients who developed VTE, in agreement with other reports [18]. Although leukemia is the most common cancer associated with VTE, the majority of our cohort (70\%) had other types of cancer. Unlike adults with brain tumors, VTE in children with brain tumors is quite low; we observed only (1.7\%) consistent with other reports $(0.64 \%-2.8 \%)$ [20-22].

A great deal of debate remains on the risk factors associated with VTE in pediatric cancer patients $[18,23,24]$. In the present study, we have identified asparaginase use concomitantly with steroids as a risk factor for VTE in B-ALL patients in-line with the published literature $[9,16,25,26]$. Although our study did not specifically look into all the risk factors associated with VTE due to its retrospective nature, larger prospective studies incorporating all potential risk factors associated with VTE are needed to solve the query.

The current study confirms previous observations that VTE is most frequently observed during chemotherapy [23]. Almost half of VTE events occurred during the first month of therapy, and two-thirds of events occurred within two months. This observation, if confirmed, potentially has important implications in designing screening and interventional strategies in terms of thromboprophylaxis for pediatric cancer patients.

Another important observation is that LMWH was effective in managing VTE in pediatric cancer patients. As all our patients with VTE were treated with Enoxaparin, only two patients (4.4\%) developed recurrent VTE events. The rate of recurrence in our study is lower than that reported in the literature (13.8\%) [19]. Anticoagulation was complicated by bleeding in only three patients (6.6\%), one minor and two major bleeding; all have recovered. LMWH was safe and effective for the management of VTE in pediatric cancer patients.

The data on thromboprophylaxis in children with cancer is scarce [15,27]. Specifically, in ALL, three studies 
have evaluated the use of LMWH at the start of asparaginase treatment and until one week after the last dose. In a prospective cohort study of 41 children with ALL, Elhasid et al. showed that LMWH might be effective [28]. Meister et al. compared the effect of anti-thrombin (AT) substitution alone versus LMWH; VTE occurred in nine (12.7\%) of 71 patients with AT substitution alone, while no VTE occurred in 41 patients with AT plus LMWH ( $\mathrm{p}=0.02)$ [29].

The authors concluded that LMWH prophylaxis was safe and effective. Harlev et al. administered LMWH only to patients with inherited thrombophilia and ALL and concluded that routine evaluation of thrombophilia followed by thromboprophylaxis to those who screen positive may benefit the at-risk patients with ALL [30]. Although thromboprophylaxis seems to be safe, the available literature is not convincing its routine use in children with cancer. However, it may be reasonable to use thromboprophylaxis in certain high-risk situations with a careful balance between risk and benefit. The Children's Oncology Group anticoagulant prophylaxis phase 3 study (ACCL 1333) is currently testing the efficacy of Apixaban for primary VTE prevention versus no anticoagulation during induction chemotherapy in children with newly diagnosed ALL treated with asparaginase [25].

Although our study has considerable limitations in terms of the retrospective design, heterogeneous group of patients and diagnoses, and the inclusion of symptomatic VTE events only, our study catches all patients with symptomatic VTE in our institution. Moreover, our study did not specifically assess all risk factors associated with VTE due to its retrospective nature and limited sample size. Our data suggest that, unlike adults where most VTE events occur in the ambulatory setting, most VTE events relate more to recent hospitalizations and chemotherapy administration. Moreover, our data show that usage of steroids concurrently with asparaginase might increase the risk of VTE, more so for leukemia patients. LMWH is safe and effective in VTE management in pediatric cancer patients. These findings set the stage for future evaluations with prospective studies to evaluate the role of thromboprophylaxis for leukemia patients receiving concurrent steroid and asparaginase during induction and consolidation chemotherapy.

\section{Conclusions}

Compared to adults, thrombosis in pediatric patients, even among those with cancer, is relatively rare. Recent hospitalization and treatment with chemotherapy are major risk factors. In addition, using steroids concurrently with asparaginase might increase the risk of VTE. Anticoagulation with LMWH is effective and safe in the treatment of thrombosis in pediatric oncology patients. Larger prospective studies incorporating all potential risk factors associated with VTE are needed to solve the query. Although thromboprophylaxis seems to be safe, the available literature is not convincing its routine use in children with cancer, and it may be reasonable to use thromboprophylaxis in certain high-risk situations with careful balance between risk and benefit.

\section{Additional Information \\ Disclosures}

Human subjects: Consent was obtained or waived by all participants in this study. King Hussein Cancer Center (KHCC) IRB Committee issued approval 18-KHCC-107. This study was approved by the Institutional Review Board (IRB) at King Hussein Cancer Center, Amman, Jordan (approval number: 18-KHCC-107). Animal subjects: All authors have confirmed that this study did not involve animal subjects or tissue. Conflicts of interest: In compliance with the ICMJE uniform disclosure form, all authors declare the following: Payment/services info: All authors have declared that no financial support was received from any organization for the submitted work. Financial relationships: All authors have declared that they have no financial relationships at present or within the previous three years with any organizations that might have an interest in the submitted work. Other relationships: All authors have declared that there are no other relationships or activities that could appear to have influenced the submitted work.

\section{References}

1. Unal S, Varan A, Yalçin B, Büyükpamukçu M, Gürgey A: Evaluation of thrombotic children with malignancy . Ann Hematol. 2005, 84:395-9. 10.1007/s00277-005-1004-x

2. Noble S, Pasi J: Epidemiology and pathophysiology of cancer-associated thrombosis. Br J Cancer. 2010, 102:S2-9. 10.1038/sj.bjc.6605599

3. Abdel-Razeq H, Mansour A, Saadeh SS, et al.: The application of current proposed venous thromboembolism risk assessment model for ambulatory patients with cancer. Clin Appl Thromb Hemost. 2018, 24:429-33. 10.1177/1076029617692880

4. Bordbar M, Karimi M, Shakibazad N: Thrombosis in pediatric malignancy: a review and future perspectives with focus on management. Blood Coagul Fibrinolysis. 2018, 29:596-601. 10.1097/MBC.0000000000000772

5. Barg AA, Kenet G: Cancer-associated thrombosis in pediatric patients. Thromb Res. 2020, 191:S22-5. 10.1016/S0049-3848(20)30392-3

6. Levy-Mendelovich S, Barg AA, Kenet G: Thrombosis in pediatric patients with leukemia . Thromb Res. 2018, 164:S94-7. 10.1016/j.thromres.2018.01.019

7. Stine KC, Saylors RL, Saccente CS, Becton DL: Treatment of deep vein thrombosis with enoxaparin in pediatric cancer patients receiving chemotherapy. Clin Appl Thromb Hemost. 2007, 13:161-5.

$10.1177 / 1076029606298989$ 
8. Athale U: Thrombosis in pediatric cancer: identifying the risk factors to improve care . Expert Rev Hematol. 2013, 6:599-609. 10.1586/17474086.2013.842124

9. Athale UH, Chan AK: Thromboembolic complications in pediatric hematologic malignancies . Semin Thromb Hemost. 2007, 33:416-26. 10.1055/s-2007-976177

10. Lipay NV, Zmitrovich AI, Aleinikova OV: Epidemiology of venous thromboembolism in children with malignant diseases: a single-center study of the Belarusian Center for Pediatric Oncology and Hematology. Thromb Res. 2011, 128:130-4. 10.1016/j.thromres.2011.03.014

11. Monagle P, Adams M, Mahoney M, et al.: Outcome of pediatric thromboembolic disease: a report from the Canadian Childhood Thrombophilia Registry. Pediatr Res. 2000, 47:763-6. 10.1203/00006450-20000600000013

12. Jaffray J, Young G: Deep vein thrombosis in pediatric patients. Pediatr Blood Cancer. 2018, 65:3. $10.1002 /$ pbc. 26881

13. Ko RH, Thornburg CD: Venous thromboembolism in children with cancer and blood disorders . Front Pediatr. 2017, 5:12. 10.3389/fped.2017.00012

14. Guyatt GH, Akl EA, Crowther M, Gutterman DD, Schuünemann HJ, American College of Chest Physicians Antithrombotic Therapy and Prevention of Thrombosis Panel: Executive summary: Antithrombotic Therapy and Prevention of Thrombosis, 9th ed: American College of Chest Physicians Evidence-Based Clinical Practice Guidelines. Chest. 2012, 141:7S-47S. 10.1378/chest.1412S3

15. van Ommen $\mathrm{CH}$, Chan AK: Supportive care in pediatric cancer: the road to prevention of thrombosis . Semin Thromb Hemost. 2014, 40:371-81. 10.1055/s-0034-1370795

16. Caruso V, Iacoviello L, Di Castelnuovo A, Storti S, Mariani G, de Gaetano G, Donati MB: Thrombotic complications in childhood acute lymphoblastic leukemia: a meta-analysis of 17 prospective studies comprising 1752 pediatric patients. Blood. 2006, 108:2216-22. 10.1182/blood-2006-04-015511

17. Wermes C, von Depka Prondzinski M, Lichtinghagen R, Barthels M, Welte K, Sykora KW: Clinical relevance of genetic risk factors for thrombosis in paediatric oncology patients with central venous catheters. Eur J Pediatr. 1999, 158:S143-6. 10.1007/p100014341

18. Athale U, Siciliano S, Thabane L, et al.: Epidemiology and clinical risk factors predisposing to thromboembolism in children with cancer. Pediatr Blood Cancer. 2008, 51:792-7. 10.1002/pbc.21734

19. Asyyed Z, MacDonald T, Digout C, Kulkarni K: Incidence and characteristics of venous thrombotic events in pediatric cancer patients: a 20-year experience in the Maritimes, Canada. Pediatr Hematol Oncol. 2017, 34:90-9. 10.1080/08880018.2017.1319450

20. Tabori U, Beni-Adani L, Dvir R, et al.: Risk of venous thromboembolism in pediatric patients with brain tumors. Pediatr Blood Cancer. 2004, 43:633-6. 10.1002/pbc.20149

21. Deitcher SR, Gajjar A, Kun L, Heideman RL: Clinically evident venous thromboembolic events in children with brain tumors. J Pediatr. 2004, 145 :848-50. 10.1016/j.jpeds.2004.05.055

22. Graham RT, Coven SL, Stanek JR, Folta A, Hollingsworth EW, Finlay JL, Kumar R: Venous thromboembolism in children with central nervous system tumors: comparison of an institutional cohort to a national administrative database. Pediatr Blood Cancer. 2021, 68:e28846. 10.1002/pbc.28846

23. Mitchell L, Lambers M, Flege S, et al.: Validation of a predictive model for identifying an increased risk for thromboembolism in children with acute lymphoblastic leukemia: results of a multicenter cohort study. Blood. 2010, 115:4999-5004. 10.1182/blood-2010-01-263012

24. Mitchell LG, Andrew M, Hanna K, et al.: A prospective cohort study determining the prevalence of thrombotic events in children with acute lymphoblastic leukemia and a central venous line who are treated with L-asparaginase: results of the Prophylactic Antithrombin Replacement in Kids with Acute Lymphoblastic Leukemia Treated with Asparaginase (PARKAA) Study. Cancer. 2003, 97:508-16. 10.1002/cncr.11042

25. Giordano P, Molinari AC, Del Vecchio GC, et al.: Prospective study of hemostatic alterations in children with acute lymphoblastic leukemia. Am J Hematol. 2010, 85:325-30. 10.1002/ajh.21665

26. Al-Aridi C, Abboud MR, Saab R, Eid D, Jeha S, Chan AK, Muwakkit SA: Thrombosis in children with acute lymphoblastic leukemia treated at a tertiary care center in Lebanon: revisiting the role of predictive models. Pediatr Hematol Oncol. 2011, 28:676-81. 10.3109/08880018.2011.578705

27. Tullius BP, Athale U, van Ommen CH, Chan AK, Palumbo IS, Balagtas JM, Subcommittee on Hemostasis and Malignancy and the Subcommittee on Pediatric/Neonatal Thrombosis and Hemostasis: The identification of at-risk patients and prevention of venous thromboembolism in pediatric cancer: guidance from the SSC of the ISTH. J Thromb Haemost. 2018, 16:175-80. 10.1111/jth.13895

28. Elhasid R, Lanir N, Sharon R, et al.: Prophylactic therapy with enoxaparin during L-asparaginase treatment in children with acute lymphoblastic leukemia. Blood Coagul Fibrinolysis. 2001, 12:367-70. 10.1097/00001721-200107000-00005

29. Meister B, Kropshofer G, Klein-Franke A, Strasak AM, Hager J, Streif W: Comparison of low-molecularweight heparin and antithrombin versus antithrombin alone for the prevention of symptomatic venous thromboembolism in children with acute lymphoblastic leukemia. Pediatr Blood Cancer. 2008, 50:298-303. $10.1002 / \mathrm{pbc} .21222$

30. Harlev D, Zaidman I, Sarig G, Ben Arush MW, Brenner B, Elhasid R: Prophylactic therapy with enoxaparin in children with acute lymphoblastic leukemia and inherited thrombophilia during L-asparaginase treatment. Thromb Res. 2010, 126:93-7. 10.1016/j.thromres.2010.04.013 